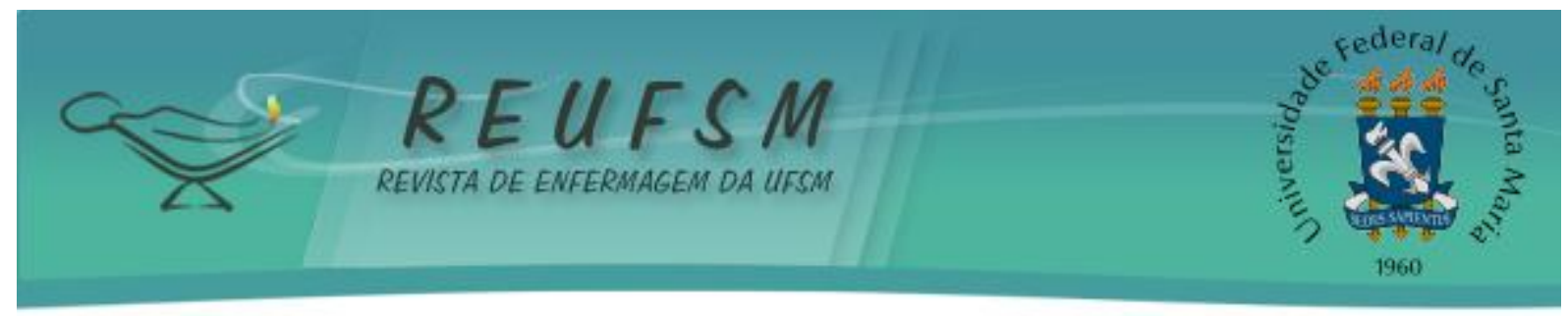

REVISÃO

\title{
PRODUÇÃO CIENTÍFICA SOBRE OS DISTÚRBIOS PSİQUICOS MENORES A PARTIR DO SELF REPORT QUESTIONNARIE
}

\author{
SCIENTIFIC PRODUCTION ABOUT MINOR PSYCHIATRIC DISORDERS FROM SELF REPORT \\ QUESTIONNARIE
}

\section{LA PRODUCCIÓN CIENTÍFICA SOBRE LOS DISTURBIOS PSÍQUICOS MENORES A PARTIR DEL SELF REPORT QUESTIONNARIE}

\author{
Juliana Petri Tavares ${ }^{1}$ \\ Carmem Lúcia Colomé Beck ${ }^{2}$ \\ Tânia Solange Bosi de Souza Magnago ${ }^{3}$ \\ Patrícia Bitencourt Toscani Greco ${ }^{4}$ \\ Francine Cassol prestes ${ }^{5}$ \\ Rosângela Marion da Silva ${ }^{6}$
}

RESUMO: Objetivo: conhecer a produção científica publicada no Brasil em bases de dados eletrônicas sobre os Distúrbios Psíquicos Menores a partir do Self Report Questionarie, bem como identificar possíveis relações entre distúrbios psíquicos menores e trabalho. Método: realizou-se um levantamento bibliográfico nas bases de dados LILACS, MEDLINE e SCIELO, nos últimos dez anos, com as palavras-chave: transtornos mentais comuns, distúrbios psíquicos menores, morbidades psiquiátricas menores, problemas psiquiátricos menores. Resultados: obteve-se 41 estudos, sendo 38 artigos científicos e três dissertações. Observa-se um aumento de publicações do ano de 2004 (2,4\%) para o de 2005 (14,6\%). 0 periódico com maior número de publicações foi o Caderno de Saúde Pública $(24,4 \%)$. Os estudos investigaram populações com idade maior ou igual a 14 anos $(48,8 \%)$; trabalhadores da saúde $(19,5 \%)$, trabalhadores de outras áreas $(19,5 \%)$ e estudantes $(12,2 \%)$. Conclusão: evidenciou-se que os distúrbios psíquicos menores estão presentes em diversas populações, de variadas regiões do país, apontando para a necessidade de novos estudos que investiguem a associação entre DPM e as condições de trabalho.

\footnotetext{
${ }^{1}$ Enfermeira. Mestranda no Programa de Pós-Graduação em Enfermagem da Universidade Federal de Santa Maria (UFSM). Membro do Grupo de Pesquisa: Trabalho, Saúde, Educação e Enfermagem do Departamento de Enfermagem da UFSM- Linha de Pesquisa Saúde do Trabalhador. Santa Maria, Rio Grande do Sul, Brasil. E-mail: jupetritavares@gmail.com

2 Enfermeira. Doutora em Enfermagem. Professor Associado II do Departamento de Enfermagem da UFSM. Membro do Grupo de Pesquisa: Trabalho, Saúde, Educação e Enfermagem da UFSM. Santa Maria, Rio Grande do Sul, Brasil. E-mail: carmembeck@gmail.com

${ }^{3}$ Enfermeira. Doutora em Enfermagem. Professor Adjunto do Departamento de Enfermagem da UFSM. Membro do Grupo de Pesquisa: Trabalho, Saúde, Educação e Enfermagem do Departamento de Enfermagem da UFSMLinha de Pesquisa Saúde do Trabalhador. Santa Maria, Rio Grande do Sul, Brasil. tmagnago@terra.com.br

${ }^{4}$ Enfermeira. Mestranda no Programa de Pós-Graduação em Enfermagem da Universidade Federal de Santa Maria (UFSM). Bolsista CAPES. Membro do Grupo de Pesquisas: Trabalho, Saúde, Educação e Enfermagem do Departamento de Enfermagem da UFSM- Linha de Pesquisa Saúde do Trabalhador. Santa Maria, Rio Grande do Sul, Brasil. E-mail: pbtoscani@hotmail.com

${ }^{5}$ Enfermeira da Clínica Renal de Santa Maria/RS. Mestranda no Programa de Pós-Graduação em Enfermagem da UFSM. Membro do Grupo de Pesquisa: Trabalho, Saúde, Educação e Enfermagem da UfSM. Santa Maria, Rio Grande do Sul, Brasil. E-mail: francinecassol@gmail.com

${ }^{6}$ Enfermeira do Hospital Universitário de Santa Maria, Mestre em Enfermagem pela UFSM. Membro do Grupo de Pesquisas: Trabalho, Saúde, Educação e Enfermagem da UFSM. Santa Maria, Rio Grande do Sul, Brasil. E-mail: cucasma@terra.com.br
} 


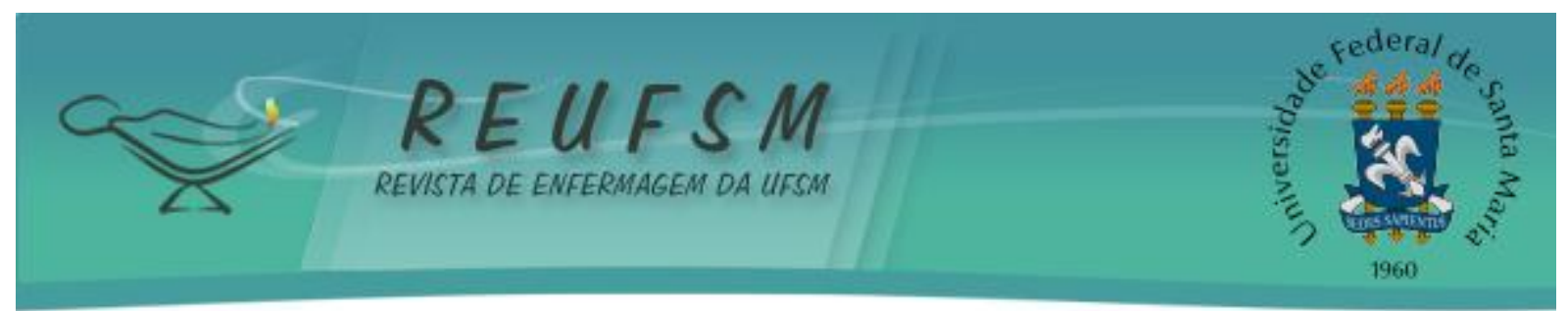

Descritores: Enfermagem; Transtornos mentais; Saúde do trabalhador; Saúde mental.

ABSTRACT: Objective: to know the scientific production published in Brazil in electronic databases on Minor Psychiatric Disorders (MPD) from the Self Report Questionnaire, and to identify possible relationships between mental disorders and minor work. Methods: We performed a literature research in the following databases LILACS, MEDLINE and SCIELO, from the last ten years, with the key words: mental disorders, minor mental disorders, minor psychiatric disorders, and minor psychiatric problems. Results: We obtained 41 studies, 38 scientific articles and three dissertations. It is observed a publication increase from 2004 (2.4\%) to 2005 (14.6\%). The journal with the largest number of publications was "Reports in Public Health" (24.4\%). The studies investigated people aged greater than or equal to 14 years (48.8\%); health workers (19.5\%), workers from other areas (19.5\%) and students (12.2\%). Conclusion: It was evidenced that minor psychiatric disorders are present in several populations from our country's different regions, pointing to the need for further studies investigating the association between MPD and working conditions.

Descriptors: Nursing; Mental disorders; Occupational health; Mental health.

RESUMEN: Objetivo: conocer la producción científica publicada en Brasil en bases de datos electrónicas acerca de los Disturbios Psíquicos Menores a partir del Self Report Questionarie, como también identificar posibles relaciones entres disturbios psíquicos menores y el trabajo. Método: se hizo un recorrido bibliográfico por las bases de datos LILACS, MEDLINE y SCIELO, en el que se consideraron estudios de los últimos diez años, con las palabras clave: transtornos mentais comuns, distúrbios psíquicos menores, morbidades psiquiátricas menores, problemas psiquiátricos menores. Resultados: se obtuvieron 41 estudios, de los cuales 38 son artículos científicos y tres, tesis de maestría. Se percibe un incremento en las publicaciones del año 2004 (el 2,4\%) al año 2005 (el 14,6\%). El periódico con mayor número de publicaciones fue el Caderno de Saúde Pública (el 24,4\%). Los estudios investigaron poblaciones con edad superior o igual a 14 años (el 48,8\%); trabajadores de la salud (el 19,5\%), trabajadores de otras áreas (el 19,5\%) y estudiantes (12,2\%). Conclusión: se evidenció que los disturbios psíquicos menores están presentes en diversas poblaciones, de diversas regiones del país, lo que señala la necesidad de nuevos estudios que investiguen la asociación entre TPM y las condiciones laborales.

Descriptores: Enfermería; Trastornos mentales; Salud laboral; Salud mental.

\section{INTRODUÇÃO}

Os distúrbios psíquicos menores (DPM) têm sido objeto de diversos estudos na última década. ${ }^{1-4}$ Parte destes estudos relaciona possíveis características do trabalho com o sofrimento psíquico e o adoecimento dos trabalhadores. As especificidades de cada contexto de trabalho podem desencadear sérios agravos à saúde física e mental dos trabalhadores, uma vez que os processos de desgaste do corpo no ambiente de trabalho são determinados, em grande parte, pelo tipo de trabalho e pela forma como essa atividade está organizada. ${ }^{5}$ Apesar dos problemas de saúde mental no trabalho serem reconhecidos, a prevenção e intervenção frente a esta problemática se constituem um grande desafio para a saúde pública. ${ }^{6}$

Dentre os problemas de saúde mental, destacamos os Distúrbios Psíquicos Menores (DPM), que designam quadros clínicos em indivíduos com sintomas de ansiedade, depressão ou somatização e que não satisfazem a todos os critérios de doença mental de acordo com a Classificação Internacional das Doenças (CID-10). ${ }^{7}$ Os 


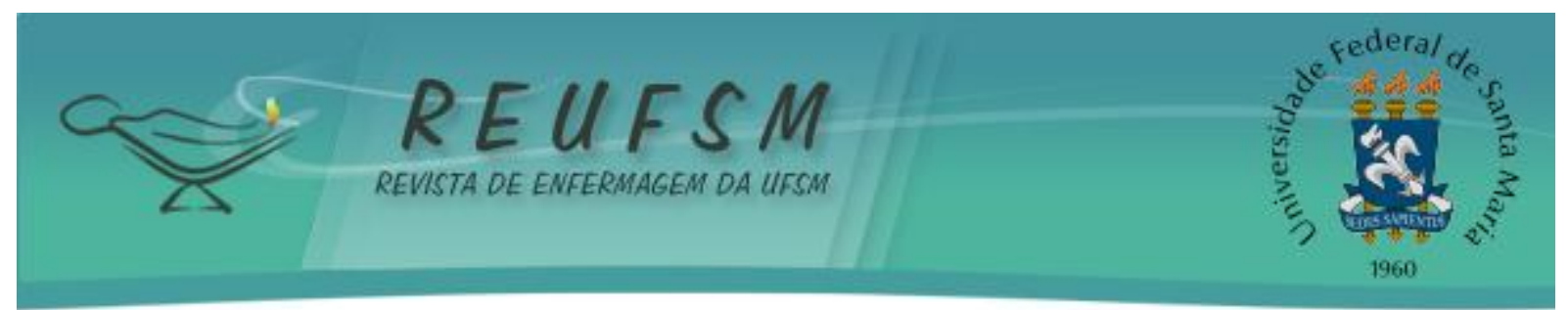

Distúrbios Psíquicos Menores são muito comuns e de difícil caracterização, sendo que a maioria dos indivíduos com tais distúrbios apresenta queixas como tristeza, ansiedade, fadiga, diminuição da concentração, preocupação somática, irritabilidade e insônia. ${ }^{8}$ Tais sintomas proporcionam incapacidade funcional comparável ou mais graves do que os quadros crônicos já bem estabelecidos. ${ }^{9}$ Diante da ausência na Classificação Internacional das Doenças (CID) de um grupo de diagnósticos de distúrbios psíquicos envolvendo os sintomas supracitados, encontrando-se diferentes denominações como, por exemplo, Morbidade Psiquiátrica Menor (MPM), Transtornos Mentais Comuns (TMC), Problemas Psiquiátricos Menores (PPM) e Distúrbios Psíquicos Menores (DPM). Assim sendo, para este estudo utilizaremos a terminologia DPM.

O Self-Report Questionnaire-20 (SRQ-20), permite a detecção precoce de sinais e sintomas de comprometimento da saúde mental, sendo recomendado, portanto, pela Organização Mundial da Saúde (OMS) como um método para identificação dos DPM. ${ }^{10}$

O SRQ foi desenvolvido como uma parte de um estudo colaborativo coordenado pela OMS com intuito de propagar os cuidados de saúde mental. Originalmente, o SRQ consistia em 25 questões, sendo 20 relacionadas a sintomas não psicóticos, quatro referentes a sintomas psicóticos e um sobre convulsões. Porém, foi detectado que as questões relacionadas aos sintomas psicóticos, não eram suficientes para detectar tais sintomas. ${ }^{11}$ A versão em português adotou os 20 primeiros itens para distúrbios não psicóticos. O SRQ-20 foi validado, no Brasil, por Mari e Williams ${ }^{11}$, revelando-se um instrumento com boa especificidade nos seus resultados. O SRQ-20 contém 20 questões sobre sintomas e problemas que tenham ocorrido nos últimos 30 dias anteriores a resposta. Cada uma das alternativas tem escore de (0) a (1), em que o escore um (1) indica que os sintomas estavam presentes no último mês e zero $(0)$ quando estavam ausentes. ${ }^{11} 0$ ponto de corte para suspeição de DPM é de seis ou mais respostas positivas para homens e de oito ou mais para mulheres. ${ }^{10}$ Alguns estudos com mulheres utilizam um ponto de corte igual a sete respostas positivas. ${ }^{2,5,12}$

Frente à relevância desta temática, apresentamos este estudo que teve por objetivo conhecer a produção científica publicada no Brasil em bases de dados eletrônicas sobre os Distúrbios Psíquicos Menores a partir do Self Report Questionarie, bem como identificar possíveis relações entre DPM e trabalho.

\section{MÉTODO}

Trata-se de uma revisão bibliográfica acerca da produção científica sobre a temática Distúrbios Psíquicos Menores (DPM). O levantamento dos dados foi realizado na Biblioteca Virtual em Saúde, no período de abril a dezembro de 2009. Para tal, utilizaramse isoladamente, pelo método integrado (entre aspas) as seguintes palavras: transtornos mentais comuns, distúrbios psíquicos menores, morbidades psiquiátricas menores, problemas psiquiátricos menores.

$\mathrm{Na}$ base de dados Literatura Latino-Americana em Ciências da Saúde (LILACS) foram encontrados 76 estudos, na Medical Literature and Retrieval System On Line (MEDLINE) 30 e na Scientific Electronic Library Online (SCIELO) 49 produções.

Obteve-se o total de 196 estudos, sendo avaliados os títulos e os resumos. Para a realização deste estudo, teve-se como critérios de inclusão: abordar a temática DPM com a utilização do SRQ-20 para sua suspeição e ter sido publicado no período de 2000 a 2009. Foram excluídos os estudos repetidos nas diferentes bases, os que não utilizaram o instrumento SRQ-20 para a suspeição de DPM; e aqueles que se referiam aos distúrbios psiquiátricos maiores, totalizando 155 estudos excluídos. 0 país de afiliação, e o idioma de 


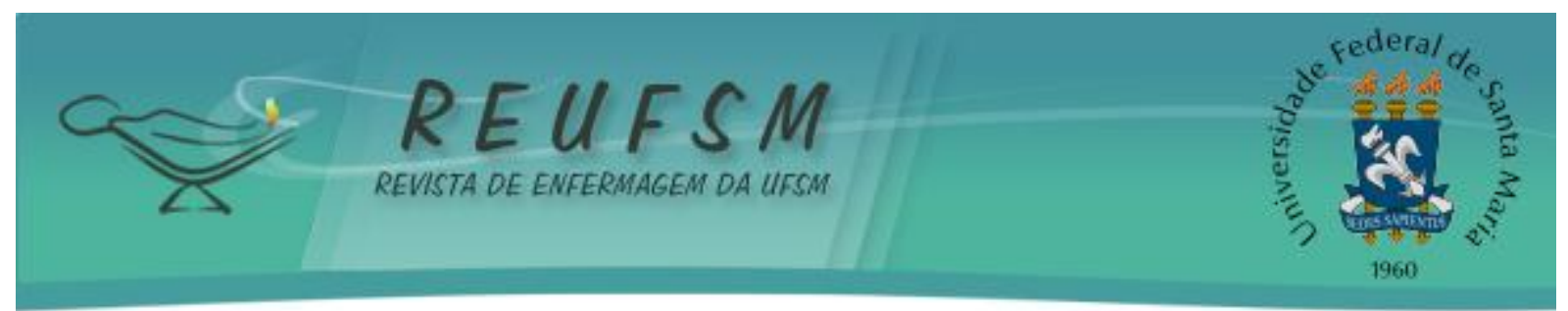

publicação não fizeram parte dos critérios de inclusão/exclusão do estudo. Portanto, foram incluídos 41 estudos, sendo 38 artigos científicos e três dissertações.

De posse dos textos na íntegra, procedeu-se a leitura crítica de todo o material, realizada a partir de uma ficha de coleta de dados em que foram registradas informações sobre a identificação das produções, objetivos, material e método, principais resultados, e possível associação entre DPM e trabalho. A partir da compreensão e análise dos dados obtidos por meio da leitura do material selecionado, evidenciaram-se os resultados descritos a seguir.

\section{RESULTADOS E DISCUSSÃO}

Foram analisadas 41 produções científicas nacionais, 38 em periódicos e três em dissertações. De acordo com a análise, observa-se que as revistas que obtiveram maior número de publicações foram a Caderno de Saúde Pública com 24,4\% $(n=10)$, a Revista de Saúde Pública com 14,6\% ( $n=6)$, o Jornal Brasileiro de Psiquiatria com 12,2\% $(n=5)$, a Revista Brasileira de Psiquiatria com 9,7\% ( $n=4)$, a Revista de Enfermagem da UERJ (Universidade Estadual do Rio de Janeiro) e a Revista da Associação Médica Brasileira com 4,8\% $(n=2)$ cada uma, sendo que as demais produções $(n=9)$ foram publicadas uma em cada periódico. Tendo em vista que as produções são da área da saúde mental e muitas se caracterizam como estudos epidemiológicos, é compreensível a seleção destas revistas para publicação. Dentre as dissertações encontradas, duas delas verificaram a relação dos distúrbios psíquicos menores com o trabalho, sendo que uma delas envolveu trabalhadores da atenção básica e a outra, docentes de uma Universidade pública. A terceira dissertação estudou a prevalência de DPM do beber problemático em pacientes com 16 anos ou mais e foi realizada no Centro de Saúde Escola (CSE) em Botucatú-SP.

Com relação ao recorte temporal estabelecido, observou-se que o ano de 2007 possui o maior número de produções com $24,4 \%(n=10)$, seguido pelo ano de 2006 com $17 \%$ $(n=7)$, os anos de 2005 e 2008 com 14,6\% ( $n=6)$ e o ano de 2009 com 12,2\% ( $n=5)$. Observouse também um acréscimo nas publicações do ano de 2004 com 2,4\% $(n=1)$ para o ano de 2005 com 14,6\% ( $n=6)$.

Essa ampliação nas publicações coincide com a aprovação da Política Nacional de Segurança e Saúde do Trabalhador (PNSST), em novembro de 2004, com vistas a possibilitar que o trabalho seja realizado em condições que contribuam para a melhoria da qualidade de vida, para a realização pessoal e social dos trabalhadores e sem prejuízo para sua saúde, integridade física e mental. ${ }^{13}$

No que se refere as regiões do País, $56,1 \%(n=23)$ foram desenvolvidos na região Nordeste, 34,1\% $(n=14)$ na região Sudeste, e 9,7\% $(n=4)$ na região Sul. Os grupos pesquisados variaram, sendo que as populações mais pesquisadas, de acordo com a Tabela 1 foram: populações não relacionadas à atividade laboral com 48,8\% ( $n=20)$; trabalhadores da saúde com 19,5\% ( $n=8)$, trabalhadores de outras áreas com 19,5\% $(n=8)$ e estudantes com $12,2 \%(n=5)$. 


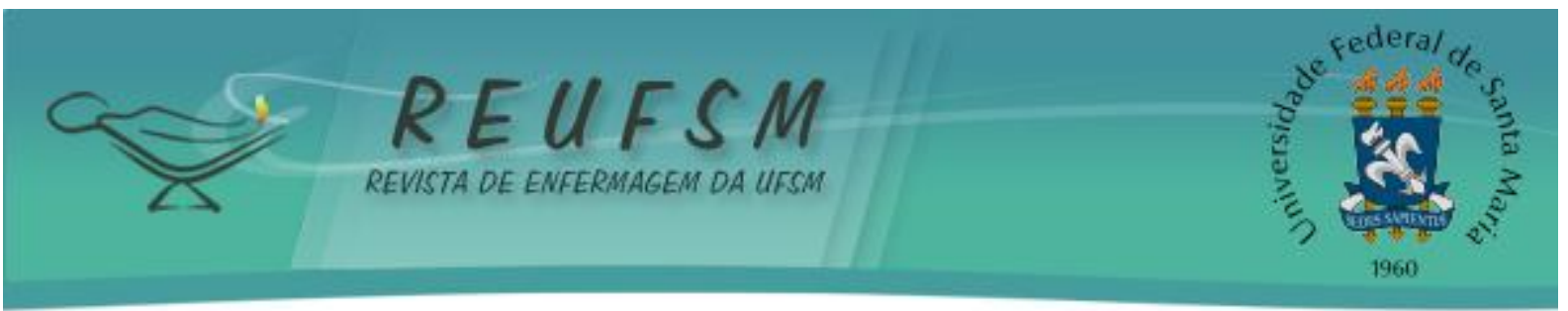

Tabela 1- Frequência de publicações brasileiras sobre Distúrbios Psíquicos Menores de acordo com a população estudada, janeiro de 2000 a dezembro de 2009. Brasil, 2009.

\begin{tabular}{l|c|c}
\hline População & $\begin{array}{c}\text { Frequência } \\
\text { (n) }\end{array}$ & $\begin{array}{c}\text { Percentual } \\
\text { (\%) }\end{array}$ \\
\hline Populações não relacionadas à atividade laboral & 13 & 31,7 \\
Pessoas com idade maior/igual a 14 anos (zona urbana, PSF) & 2 & 4,9 \\
Mulheres com idade maior/igual 15 anos & 1 & 2,4 \\
Mulheres de 45 a 65 anos & 1 & 2,4 \\
Mulheres atendidas no Ambulatório de Esterilidade & 1 & 2,4 \\
Mulheres de 15 a 49 anos atendidas na Emergência & 1 & 2,4 \\
Adultos de 40 anos ou mais & 1 & 2,4 \\
Adolescentes com idade de 15 a 18 anos & 3 & \\
\hline Trabalhadores da saúde & 3 & 7,3 \\
Trabalhadores de enfermagem & 1 & 2,3 \\
Médicos & 1 & 2,4 \\
Trabalhadores da saúde & & \\
Agentes Comunitários de Saúde (ACS) & 3 & 7,3 \\
\hline Trabalhadores de outras áreas & 1 & 2,4 \\
Professores da Pré-escola e ensino fundamental (municipal e & 1 & 2,4 \\
particular & 1 & 2,4 \\
Agentes penitenciários & 1 & 2,4 \\
Trabalhadores com 15 anos ou mais & 1 & 2,4 \\
Professores de Educação física (EF) & & \\
Docentes do ensino superior & 3 & 7,3 \\
Motorista & 1 & 2,4 \\
\hline Estudantes & & 2,4 \\
Estudantes de medicina & 1 & 100 \\
Estudantes de Educação Física, Enfermagem, Odontologia e & 41 & \\
Medicina & & \\
Estudantes do ensino médio (particular) & & \\
\hline TOTAL & & \\
\hline
\end{tabular}

As prevalências globais de DPM encontradas em todos os estudos revisados variaram de $17 \%$ a $77,3 \%$. A menor prevalência foi encontrada em uma amostra representativa da população com 14 anos ou mais de idade, residente na zona urbana de Campinas-SP. ${ }^{14}$ Já a maior prevalência foi em mulheres, de 15 a 49 anos, vítimas de violência, atendidas em um hospital de emergência na cidade de Salvador-BA. ${ }^{15}$

Dentre as populações mais estudadas estão as não relacionadas à atividade laboral, destacando-se as seguintes populações: pessoas com mais de 14 anos $(n=13)$, mulheres $(n=5)$, adultos com 40 anos ou mais $(n=1)$ e adolescentes de 15 a 18 anos $(n=1)$.

A maior parcela dos estudos refere-se a pessoas maiores de 14 anos, destacandose os usuários de PSF $(n=3)$. Dos estudos com pessoas maiores de 14 anos, $14,6 \%(n=7)$ verificaram associação positiva entre DPM e características socioeconômicas. Além das dessas associações, foi investigado questões como uso de psicofármacos $(n=2)$, propriedades psicométricas do SRQ-20 $(n=1)$, apoio social $(n=1)$, terapias medicinais integrativas e complementares $(n=1)$, necessidades de atendimento em saúde mental $(n=1)$.

Uma parcela $(12,2 \%)$ dos estudos avaliou os DPM em mulheres $(n=5)$. Cada vez mais emergem estudos relacionados a mulheres, uma vez que ao contrário do que ocorre com os homens, a inserção feminina no mercado de trabalho é limitada por suas responsabilidades domésticas e familiares, tendo o emprego que ser adaptado a estas funções. ${ }^{16}$ 


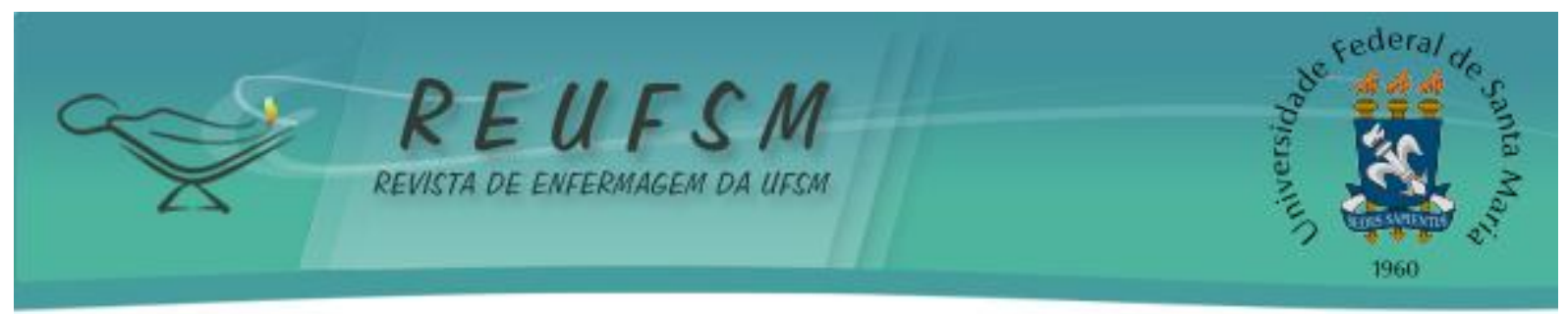

A elevada prevalência de DPM em mulheres violentadas evidencia a necessidade de medidas preventivas e a formulação de políticas de saúde que estimulem a integralidade da assistência às mulheres. Nas duas últimas décadas têm sido criados serviços, a exemplo de delegacias especiais e centros de referência para o atendimento às vítimas de agressão, destacando-se que estes serviços precisam estar articulados em rede, envolvendo as diferentes áreas do conhecimento e de atuação dos trabalhadores da saúde. ${ }^{15}$

Dos 41 estudos revisados, 16 deles estão relacionados ao exercício laboral. Desses, a maior prevalência $(55,9 \%)$ está relacionada a professores da rede pública (municipal) de ensino. ${ }^{17}$

A alta prevalência de DPM em professores evidencia uma situação preocupante e a necessidade de voltar o olhar para essa população. No Brasil, pouco se tem avaliado o reflexo do trabalho na saúde desses trabalhadores, sendo realizado alguns estudos principalmente na região Nordeste. ${ }^{17}$ Essa classe trabalhadora parece apresentar alguns riscos subjetivos, não se devendo subestimar os sinais de exaustão, os quais interferem tanto na sua saúde física e emocional quanto na atuação profissional dos docentes. ${ }^{17}$

Dos 16 estudos relacionados ao exercício laboral, nove (três com professores, três com trabalhadores de enfermagem, dois com médicos e um com profissionais da saúde) relacionam os DPM com a demanda psicológica e o controle sobre o trabalho, modelo Demanda-Controle ("Demand-Control Model" ou "Job Strain") proposto por Karasek. ${ }^{18}$ Tal modelo investiga duas dimensões psicossociais no ambiente de trabalho - demandas psicológicas e controle do trabalhador sobre o processo de trabalho e o risco de adoecimento do trabalhador. ${ }^{19}$ Desses oito estudos, em sete há uma maior chance de DPM na alta exigência no trabalho (alta demanda e baixo controle) comparado ao grupo de baixa exigência (baixa demanda e alto controle) e dois deles retrata que estava estatisticamente associado com as características do conteúdo do trabalho. ${ }^{20}$

Quanto aos outros sete estudos que relacionam as questões laborais com os DPM, cinco (um com trabalhadores informais, um com professores de educação física, um com agentes penitenciários, um com donas-de-casa, um com médicos) o relacionam com as condições de trabalho, um com esgotamento profissional em Agentes Comunitários de Saúde e um Hipertensão Arterial Sistêmica em motoristas.

As condições de trabalho interferem diretamente na saúde dos trabalhadores. Dessa forma, estudo que descreveu as condições de trabalho e os principais problemas de saúde referidos por médicos, aponta que o cenário laboral desses trabalhadores é bastante desfavorável, devido a sobrecarga de trabalho, especialmente em atividades de plantão; condições de trabalho precárias, regidas preferencialmente pela remuneração por procedimento, baixa remuneração e elevada frequência de queixas físicas e psíquicas. ${ }^{21}$

Foram encontrados cinco estudos com estudantes (três do curso de medicina, um dos cursos de enfermagem, odontologia, educação física e medicina e um com estudantes de um colégio particular). Verificou-se a maior prevalência dos DPM $(45,7 \%)$ em estudantes de um colégio particular, evidenciando-se uma associação positiva entre DPM e o sexo feminino. ${ }^{22}$

A identificação das dificuldades vivenciadas pelos alunos ao longo de cada etapa do curso pode ser indicativa da necessidade de desenvolvimento de estratégias de enfrentamento das dificuldades e de prevenção, por meio de projetos contínuos que sensibilizem os estudantes para os riscos dos transtornos psíquicos e disfunções profissionais, os quais podem interferir no bem-estar dos alunos, de seus familiares e dos pacientes sob sua responsabilidade. ${ }^{23}$ 


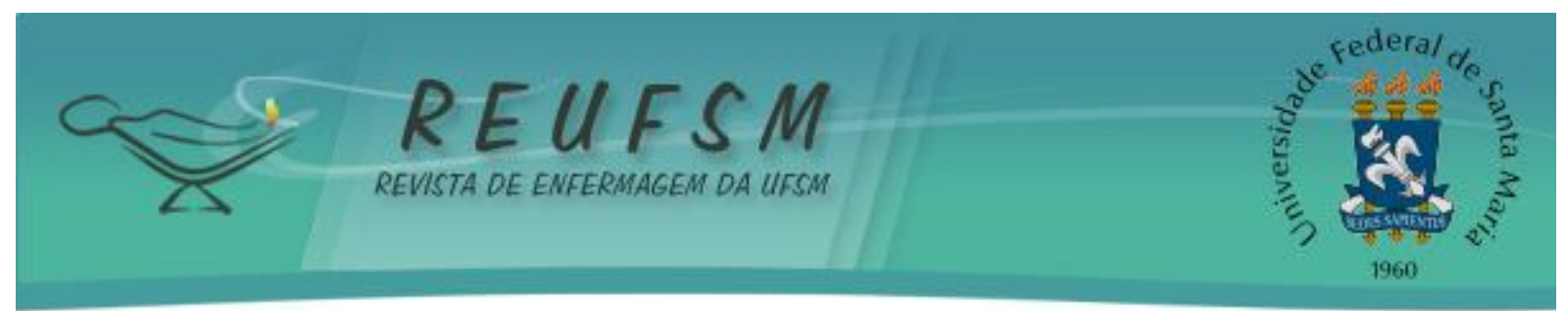

Assim, o conhecimento acerca das publicações brasileiras sobre DPM permitiu identificar estudos com diferentes grupos, o que evidencia o interesse dos pesquisadores em relação à saúde mental da população.

\section{CONCLUSÕES}

A análise das produções revisadas nesta pesquisa confirmou que os Distúrbios Psíquicos Menores estão presentes em diversas populações de variadas regiões do país sendo, portanto, um problema de saúde pública. A maior parte da produção foi identificada no ano de 2007 e o Nordeste foi a região do Brasil que mais produziu sobre o assunto.

Os resultados desta pesquisa apontam para a necessidade de melhoria nas condições e organização do trabalho dos docentes, trabalhadores de enfermagem e médicos. Também evidenciam a importância da realização de estudos que investiguem a associação entre DPM e condições de trabalho em diferentes populações. Ainda mais, fornecem subsídios para que sejam identificados os riscos e planejadas estratégias na perspectiva de colaborar para a qualidade de vida das pessoas e, principalmente, para a saúde do trabalhador.

É necessário considerar as possíveis limitações deste estudo como cortes transversais em que não se pode relacionar causa e efeito, além do fato de que os escores Self Reporting Questionnaire-20 (SRQ-20) serem apenas sugestivos de sofrimento psíquico ou de Distúrbios Psíquicos Menores.

Além disso, destaca-se a escassez de descritores específicos utilizados para caracterizar a temática Distúrbios Psíquicos Menores, o que dificulta e torna demorada a procura dos estudos, bem como os possíveis erros de classificação e seleção dos artigos. Observou-se também que alguns resumos não são estruturados, dificultando o acesso a essas publicações.

Os DPM, considerados um problema de saúde mental e por ser de difícil caracterização, precisam ser melhor investigados principalmente em docentes uma vez que fatores relacionados às condições e sobrecarga de trabalho somados ao contexto de vida dessa categoria profissional podem comprometer, a longo prazo, a saúde física e mental dessas pessoas. Estudar DPM em docentes é uma necessidade atual, pois a não identificação desses distúrbios pode comprometer tanto a qualidade do ensino quanto a qualidade de vida desses trabalhadores.

\section{REFERÊNCIAS}

1. Faria NMX, Facchini LA, Fassa AG, Tomasi E. Estudo sobre saúde mental de agricultores da Serra Gaúcha (Brasil). Rev. Saúde Pública.1999; 33(4): 391-400.

2. Araújo TM, Aquino E, Menezes G, Santos CO, Aguiar L. Aspectos Psicossociais do trabalho e distúrbios psíquicos entre trabalhadores da enfermagem. Rev. Saúde Pública. 2003; 37(4): 24-33.

3. Lurdemir $A B$. Associação dos transtornos mentais comuns com a informalidade das relações de trabalho. J. Bras. Psiquiatr. 2005; 54(3):198-204.

4. Porto LA, Carvalho FM, Oliveira NF, Silvany Neto AM, Araújo TM, Reis EJFB, et al. Associação entre distúrbios psiquiátricos e aspectos psicossociais do trabalho de professores. Rev. de Saúde Pública. 2006; 40(5): 818-26. 


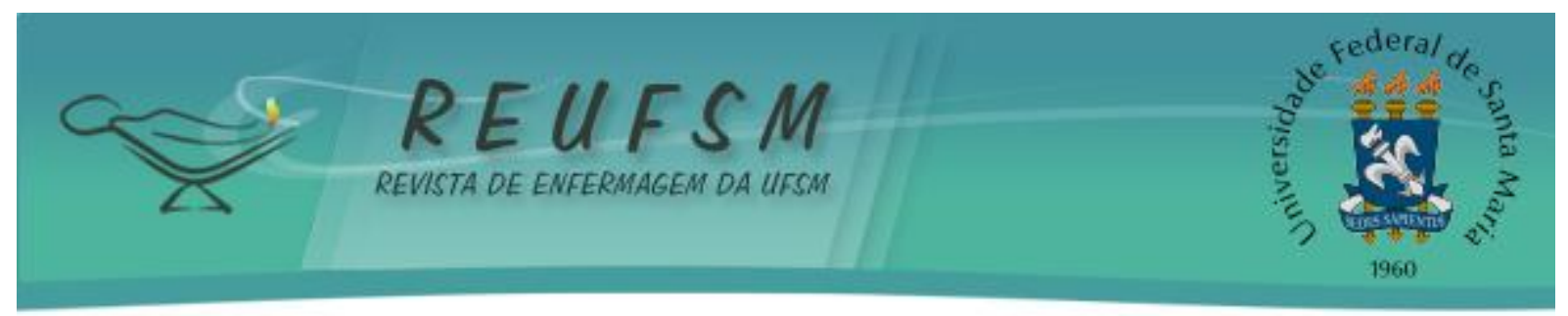

5. Delcor NS, Araújo TM, Reis EJFB, Porto LA, Carvalho FM, Silva MO, et al. Condições de trabalho e saúde dos professores da rede particular de ensino de Vitória da Conquista, Bahia, Brasil. Cad. saúde pública. 2004; 20(1):187-196.

6. Sato L, Bernardo MH. Saúde mental e trabalho: os problemas que persistem. Ciênc. Saúde Coletiva. 2005; 10( 4): 869-78.

7. Cerchiari EAN, Caetano D, Faccenda O. Prevalência de Transtornos Mentais Menores em Estudantes Universitários. Estud. psicol. 2005;10(3): 413-20.

8. Kac G, Silveira EA, Oliveira LC, Mari JJ. Fatores relacionados à prevalência de morbidades psiquiátricas menores em mulheres selecionadas em um Centro de Saúde no Rio de Janeiro, Brasil. Cad. Saúde Pública. 2006; 22 (5): 999-1007.

9. Maragno L,Goldbaum M, Gianini RJ, Novaes Hillegonda MD, César CLG et al. Prevalência de transtornos mentais comuns em populações atendidas pelo Programa Saúde da Família (QUALIS) no Município de São Paulo, Brasil. Cad. Saúde Pública. 2006; 22(8): 1639-48.

10. Mari JJ, Williams P. A validity study of a psychiatric screening questionnaire (SQR-20) in primary care in the city of São Paulo. Brit. Jour. of Psych.1986;148: 23-26.

11. WHO MP. A user's guide to the self reporting questionnarie (SRQ): Division of Mental Health World Health Organization Geneva.1994.

12. Araújo TM. Trabalho e distúrbios psíquicos em mulheres trabalhadoras de enfermagem. 1999. 210f. Tese (Doutorado em Saúde Pública) - Instituto de saúde Coletiva, Universidade Federal da Bahia, Salvador (BA).

13. Grupo de Trabalho Interministerial MPS/MS/TEM. Política Nacional de Segurança e Saúde do Trabalhador (PNSST). Brasília: GTI, 2004.

14. Marin-Leon L, Oliveira HB , MBA, Dalgalarrondo P, Botega NJ. Social inequality and common mental disorders. Rev. Bras. Psiquiatr. 2007; 29(3): 250-253.

15. Silva IV, Aquino EM. Patterns of minor psychiatric disorders among battered women treated at an emergency care unit. Cad. Saúde Pública. 2008; 24(9): 2103- 2114 .

16. Lurdermir AB. Inserção produtiva, gênero e saúde mental. Cad. Saúde Pública. 2000; 16(3): 647-59.

17. Reis EJFB. et al. Trabalho e distúrbios psíquicos em professores da rede municipal de Vitória da Conquista, Bahia, Brasil. Cad. Saúde Pública. 2005; 21(5): 1480-1490.

18. Karasek RA, Theörell T. Healthy work-stress, productivity, and the reconstruction of working life. New York: Basic Books; 1990.

19. Alves MGM, Chor D, Faerstein E, Lopes CS, Werneck GL. Versão resumida da "job stress scale” adaptação para o português. Rev. Saúde Pública. 2004; 38(2): 164-71.

20. Braga LC. Condições de trabalho e saúde dos profissionais da rede básica de saúde de Botucatu-SP. 2007. 142 f. Dissertação (Mestrado em Medicina). Universidade Estadual Paulista. Botucatu (SP).

21. Nascimento SCL. Condições de trabalho e saúde dos médicos em Salvador, Brasil. Rev. Assoc. Med. Bras. 2006; 52(2)97-102.

22. Rocha THR. Ribeiro JEC, Pereira GA, Aveiro CC, Silva LCA. Sintomas depressivos em adolescentes de um colégio particular. Psico USF. 2006; 11(1); 95-102. 


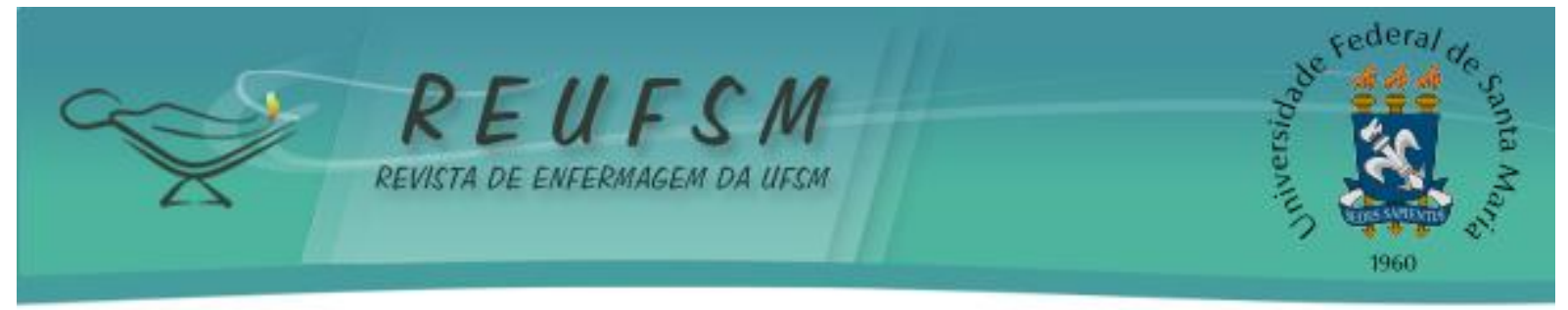

23. Amaral GF, Gomide LMP, Batista MP, Píccolo PP, Teles TBG, Oliveira PM et al . Sintomas depressivos em acadêmicos de medicina da Universidade Federal de Goiás: um estudo de prevalência. Rev. psiquiatr. Rio Gd. Sul. 2008 ; 30(2): 124-130.

\section{ANEXO}

Quadro 1: Relação dos estudos encontrados no período de janeiro de 2000 a dezembro de 2009.

\begin{tabular}{|c|}
\hline $\begin{array}{l}\text { REFERÊNCIAS DOS ARTIGOS INCLUÍDOS NO ESTUDO } \\
\text { nsercäo produtiva, gênero e saúde mental Cad. de Saúde }\end{array}$ \\
\hline $\begin{array}{l}\text { 1-Ludermir A B. Inserçäo produtiva, gênero e saúde mental. Cad. de } \\
\text { Pública.2000;16(3): 647-659. }\end{array}$ \\
\hline $\begin{array}{l}\text { 2-Wernick R. Condições de saúde e trabalho dos docentes da Universidade Federal } \\
\text { da Bahia. 2000. } 119 \text { f. Dissertação (Mestrado em Saúde Coletiva) - Universidade } \\
\text { Federal da Bahia, Salvador. }\end{array}$ \\
\hline $\begin{array}{l}\text { 3-Fernandes RCP, Silvany Neto, AM, Sena GM, Leal AS, Carneiro CAP, Costa FPM. } \\
\text { Trabalho e cárcere: um estudo com agentes penitenciários da Região Metropolitana } \\
\text { de Salvador, Brasil. Cad. Saúde Pública. } 2002 ; 18(3) \text {. }\end{array}$ \\
\hline $\begin{array}{l}\text { 4-Costa JSD, Menezes AMB, Olinto MTA, Gigante DP, Macedo S, Britto MAP, et al. } \\
\text { Prevalência de distúrbios psiquiátricos menores na cidade de Pelotas, RS. Revista } \\
\text { Brasileira de Epidemiologia. } 2002 ; 5(2): 164-173 \text {. }\end{array}$ \\
\hline $\begin{array}{l}\text { 5-Ludermir A B, Filho D A M. Condições de vida e estrutura ocupacional associadas a } \\
\text { transtornos mentais comuns. Rev. Saúde Pública. } 2002 ; 36(2): 213-21 .\end{array}$ \\
\hline $\begin{array}{l}\text { 6-Araújo TM, Aquino E, Menezes G, Santos CO, Aguiar L. Aspectos psicossociais do } \\
\text { trabalho e distúrbios psíquicos entre trabalhadoras de enfermagem. Rev. Saúde } \\
\text { Pública.2003;37(4): 424-433. }\end{array}$ \\
\hline $\begin{array}{l}\text { 7- Delcor NS, Araújo TM, Reis EJFB, Porto LA, Carvalho FM, Silva MO, et al. } \\
\text { Condições de trabalho e saúde dos professores da rede particular de ensino de } \\
\text { Vitória da Conquista, Bahia, Brasil. Cad. saúde pública. 2004; 20(1):187-196. }\end{array}$ \\
\hline $\begin{array}{l}\text { 8- Simioni L. Estudo de prevalência de morbilidade psiquiátrica e beber } \\
\text { problemático em um serviço de atenção básica à saúde. 2005. } 115 \text { f. Dissertação } \\
\text { (Mestrado em Medicina) - Universidade Estadual Paulista. Botucatu. }\end{array}$ \\
\hline $\begin{array}{l}\text { 9-Ludermir AB. Associação dos transtornos mentais comuns com a informalidade das } \\
\text { relações de trabalho. J. bras. psiquiatr.2005 54(3): 198-204. }\end{array}$ \\
\hline $\begin{array}{l}\text { 10-Araújo TM, Pinho OS, Almeida MMG. Prevalência de transtornos mentais comuns } \\
\text { em mulheres e sua relação com as características sociodemográficas e o trabalho } \\
\text { doméstico. Rev. Bras. Saúde Matern. Infant. 2005; } 5(3): 337-348 \text {. }\end{array}$ \\
\hline $\begin{array}{l}\text { 11-Facundes VLD, Ludermir A. B. Common mental disorders among health care } \\
\text { students. Rev. Bras. Psiquiatr. São Paulo.2005 27(3): 194-200. }\end{array}$ \\
\hline $\begin{array}{l}\text { 12-Costa AG, Ludermir A B. Transtornos mentais comuns e apoio social: estudo em } \\
\text { comunidade rural da Zona da Mata de Pernambuco, Brasil. Cad Saúde Pública. Rio de } \\
\text { Janeiro.2005 21(1): } 73-79 \text {. }\end{array}$ \\
\hline $\begin{array}{l}\text { 13- Reis EJFB, Carvalho FM, Araújo TM, Porto LA, Silvany Neto AM. Trabalho e } \\
\text { distúrbios psíquicos em professores da rede municipal de Vitória da Conquista, } \\
\text { Bahia, Brasil. Cad. Saúde Pública. 2005; 21(5): 1480-1490. }\end{array}$ \\
\hline $\begin{array}{l}\text { 14-Rocha THR. Ribeiro JEC, Pereira GA, Aveiro CC, Silva LCA. Sintomas depressivos } \\
\text { em adolescentes de um colégio particular. Psico USF. 2006; 11(1); 95-102. }\end{array}$ \\
\hline $\begin{array}{l}\text { 15-Lima MCP, Domingues M S. Cerqueira ATAR. Prevalência e fatores de risco para } \\
\text { transtornos mentais comuns entre estudantes de medicina. Rev. saúde pública. } \\
2006 ; 40(6): 1035-1041 .\end{array}$ \\
\hline \\
\hline
\end{tabular}




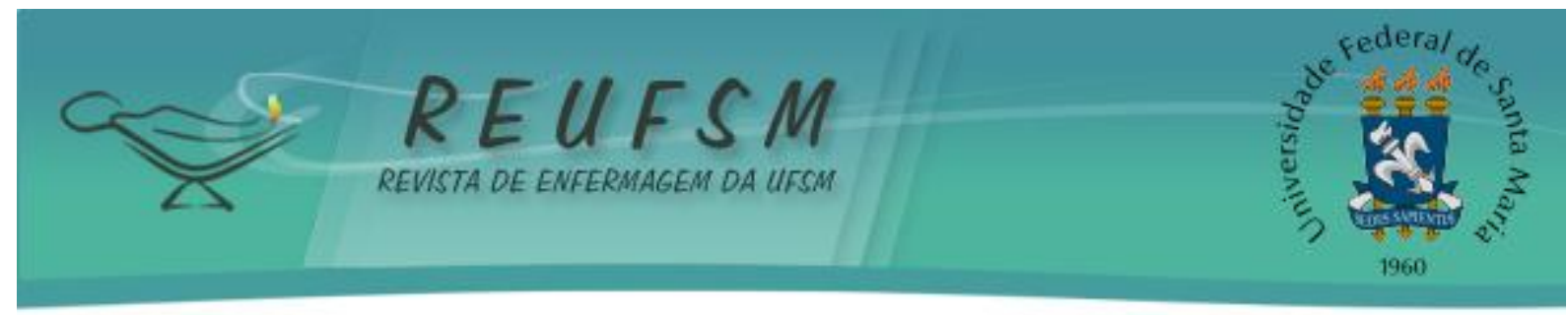

comuns em mulheres: estudo comparativo entre donas-de-casa e trabalhadoras.

Rev. Enferm. UERJ.2006; 14( 2): 260-269.

17-Maragno L, Goldbaum M, Gianini RJ, Novaes Hillegonda MD, César CLG.

Prevalência de transtornos mentais comuns em populações atendidas pelo Programa Saúde da Família (QUALIS) no Município de São Paulo, Brasil. Cad. Saúde Pública. 2006; 22(8): 1639-48.

18-Nascimento SCL. Condições de trabalho e saúde dos médicos em Salvador, Brasil. Rev. Assoc. Med. Bras. 2006; 52(2)97-102.

19- Nascimento SCL, Condições de trabalho e saúde mental dos médicos de Salvador, Bahia, Brasil. Cad. saúde pública. 2006; 22( 1): 131-140.

20-Porto LA, Carvalho FM, Oliveira NF, Silvany Neto AM, Araújo TM, Reis EJFB, et al,. Associação entre distúrbios psíquicos e aspectos psicossociais do trabalho de professores. Rev. Saúde Pública. 2006;40(5): 818-26.

21-Araújo TM, Carmo Júnior JJ, Almeida MMG, Pinho PS. Prática de atividades de lazer e morbidade psíquica em residentes de áreas urbanas. Rev. baiana saúde pública. 2007;31( 2): 294-310.

22-Pinho OS, Araújo TM. Trabalho de enfermagem em uma unidade de emergência hospitalar e transtornos mentais. Rev. enferm. UERJ. 2007;15( 3): 329-336.

23-Braga LC. Condições de trabalho e saúde dos profissionais da rede básica de saúde de Botucatu-SP. 2007. 142 f. Dissertação (Mestrado em Medicina).

Universidade Estadual Paulista. Botucatu.

24-Almeida AM, Godinho TM, Bitencourt AGV, Teles MS, Silva AS, Fonseca DC, et al. Common mental disorders among medical students. J. bras. psiquiatr. 2007; 56( 4): 245-251.

25-Galvão L L L F, Farias MCS, Azevedo PRM, Vilar MJP, Azevedo GD. Prevalência de transtornos mentais comuns e avaliação da qualidade de vida no climatério. Rev. Assoc. Med. Bras. 2007; 53( 5): 414-420.

26-Marin-León L, Oliveira HB, Barros MBA, Dalgalarrondo P, Botega NJ. Social inequality and common mental disorders. Rev. bras. psiquiatr.2007; 29( 3): 250-253. 27-Pinheiro KAT Horta BL, Pinheiro RT, Horta LL, Terres NG, Silva RA. Common mental disorders in adolescents: a population based cross-sectional study. Rev. Bras. Psiquiatr.200729(3): 241-245.

28-Cabana MCFL, Ludermir AB, Silva ER, Ferreira MLL, Pinto MER .. Transtornos mentais comuns em médicos e seu cotidiano de trabalho. J. bras. psiquiatr.2007;56( 1): 33-40.

29-Bandeira M, Freitas LC, Filho JGC. Avaliação da ocorrência de transtornos mentais comuns em usuários do Programa de Saúde da Família. J. bras.

psiquiatr.2007 56(1): 41-47.

30- Avanci JQ, Assis SG, Oliveira RVC, Ferreira RM, Pesce RP. Fatores associados aos problemas de saúde mental em adolescentes. Psicol. teor. pesqui.2007 23(3): 287294.

31-Rodrigues-Neto JF, Figueiredo MFS, Faria AAS, Fagundes M. Transtornos mentais comuns e o uso de práticas de medicina complementar e alternativa: estudo de base populacional. J. bras. psiquiatr.2008;57( 4): 233-239.

32-Silva ATC da, Menezes PR. Esgotamento profissional e transtornos mentais comuns em agentes comunitários de saúde. Rev Saúde Pública. 2008; 42(5): 921-9.

33-Lima MCP, Menezes PR, Carandina L, Cesar CLG, Barros MBA, Goldbaum, M. Transtornos mentais comuns e uso de psicofármacos: impacto das condições socioeconômicas. Rev. saúde pública, v. 42, n. 4, p. 717-723, ago, 2008. 


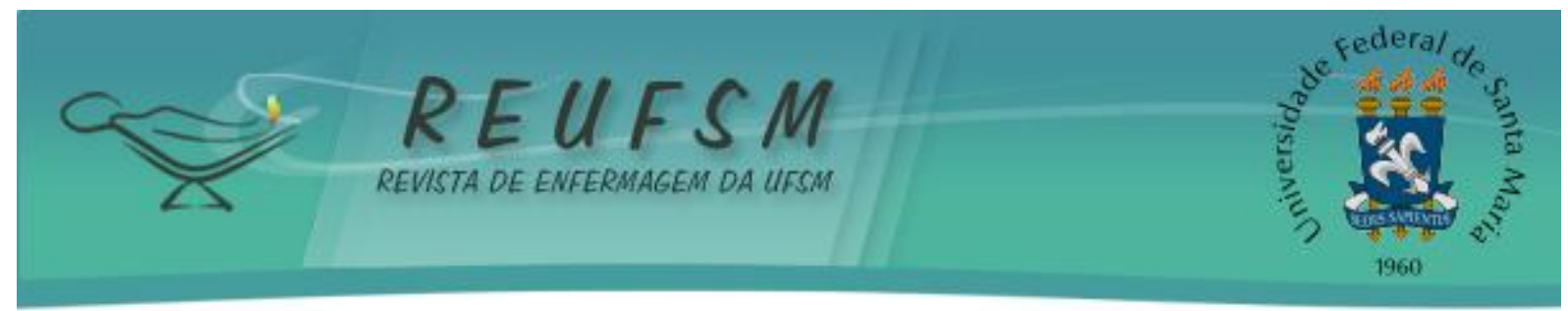

34-Gianini RJ, Carvalho TC, Anjos RMP, Pinto PLS, Maluf ME, Lanza LB, et al. Prática de rastreamento no cenário do Programa Saúde da Família em Sorocaba (SP). Rev. bras. educ. méd. 2008; 32(1):15-22.

35-Cunha MCV. Carvalho JA, Albuquerque RM, Ludermir AB, Novaes $M$, et al. Infertilidade: associação com transtornos mentais comuns e a importância do apoio social. Rev. psiquiatr. Rio Gd. Sul.2008; 30( 3): 201-210.

36-Silva IV, Aquino E M. Padrão de distúrbios psíquicos menores em mulheres vítimas de violência atendidas em uma unidade de urgência e emergência. Cad. saúde pública.2008; 24(9) 2103-2114.

37-Santos KOB, Araújo T M, Oliveira N F. Estrutura fatorial e consistência interna do Self-Reporting Questionnaire (SRQ-20) em população urbana. Cad. saúde pública.2009 25(1): 214-222.

38-Coelh FMC, Pinheiro RT, Horta BL, Magalhães PVS, Garcias CMM, Vilva CV.Transtornos mentais comuns e enfermidades crônicas em adultos: estudo de base populacional. Cad. saúde pública. 2009; 25(1): 59-67.

39-Kirchhof ALC. Magnago TSBSouza, Camponogara S, Griep RH, Tavares JP, Prestes FC, et al. Condições de trabalho e características sócio-demográficas relacionadas à presença de distúrbios psíquicos menores em trabalhadores de enfermagem. Texto contexto-enferm.2009; 18(2): 215-223.

40- Palma A, Mattos UAO, Almeida MN, Oliveira GEMC . Nível de ruído no ambiente de trabalho do professor de educação física em aulas de ciclismo indoor. Rev. saúde pública.2009;43( 2):345-351.

41- Cavagioni LC, Pierin A M G, Batista K M, Bianchi E R F, Costa A L S. Agravos à saúde, hipertensão arterial e predisposição ao estresse em motoristas de caminhão. Rev. Esc. Enferm. USP.2009; 43 (spe2): 1267-1271.

Data de recebimento:08/10/2010

Data de aceite:18/11/2010

Contato com autora responsável: Juliana Petri Tavares

E-mail: jupetritavares@gmail.com 